\title{
Extrusion of Polyethylene Single Crystals
}

\author{
Tetsuo Kanamoto, ${ }^{*}$ Edward S. Sherman, and Roger S. Porter \\ Materials Research Laboratory, Polymer Science and Engineering Department, \\ University of Massachusetts, Amherst, Massachusetts 01003, U.S.A.
}

(Received December 26, 1978)

\begin{abstract}
The solid (crystalline)-state extrusion of unoriented single-crystal aggregates and oriented single-crystal mats of high-density polyethylene (HDPE) has been studied. Irrespective of the initial state of aggregation, uniform, transparent, highly oriented and high modulus fibers and films were produced. Single crystal aggregates extruded at a rate faster than melt-crystallized spherulitic morphology. Surface cracks were observed but shear fracture seldom occurred on singlecrystal extrudates. The maximum attainable extrusion draw ratio (EDR) and hence the modulus was higher for the extrusion of single crystals than for spherulitic samples. Thus, the initial morphology had a marked effect on the extrusion of HDPE but no appreciable effect on the properties (birefringence, modulus, and transparency) of the resultant extrudates measured as a function of EDR. These results clearly show that intercrystalline networks and entanglements of the original sample have a remarkable effect on extrusion but are not important in achieving efficient draw as evaluated by the properties as a function of EDR. Moreover, the results indicate that interfacial friction-adhesion between crystalline lamellae is the primary deformation mechanism.

KEY WORDS Solid State Extrusion / High Density Polyethylene /

Morphology / Single Crystals / Efficiency of Draw / Deformation

Mechanism/
\end{abstract}

During the past several years, the mechanism of solid (crystalline)-state extrusion of semicrystalline polymers, and the consequent structure and properties of extrudates have been extensively studied in this and other laboratories. ${ }^{1-11}$ However, little effort has been made to determine the effect of initial morphology on the crystalline state extrusion. This variable is known to have an important effect on the cold drawing of high-density polyethylene (HDPE) of initial spherulitic morphology. ${ }^{12,13}$ Recently, Mead and Porter ${ }^{14}$ extruded HDPE having a range of initial morphologies prepared at different temperatures and pressures. They reported that a morphology intermediate between the quenched and extended-chain crystals gave the highest extrusion draw ratio (EDR) and hence the highest Young's modulus. This work is limited to melt-crystallized morphologies. A limiting state for semicrystalline polymers is the single-crystal morphology as grown

\footnotetext{
* Department of Chemistry, Science University of Tokyo, Tokyo 162, Japan.
}

from dilute solution. Cold drawing of oriented single-crystal mats of HDPE has been extensively studied by Ishikawa, et al. ${ }^{15,16}$ Statton, ${ }^{17}$ and Barham, et al. ${ }^{18}$ However, the deformation mode for crystalline-state extrusion is more versatile and complex than in conventional cold drawing. ${ }^{19}$

In this work, both unoriented single-crystal aggregates and oriented single crystal mats of HDPE were extruded at temperatures of 70 to $110^{\circ} \mathrm{C}$, i.e., below the ambient melting point $\sim 133^{\circ} \mathrm{C}$ and up to extrusion draw ratio, EDR, of 45. Irrespective of the initial state of single-crystal aggregation, transparent, highly birefringent and high modulus fibers and films were produced. The main purpose of this paper is thus to report the characteristics of the extrusion behavior of unoriented single-crystal aggregates of HDPE. The birefringence and tensile modulus of the extrudates are also reported as a function of EDR defined as the ratio of crosssectional area change of the materials. 


\section{EXPERIMENTAL}

\section{Preparation of Solution-Grown Crystals}

About $25 \mathrm{~g}$ of linear high-density polyethylene, HDPE, (du Pont Alathon 7050, $M_{w}=58,000$, $M_{w} / M_{n}=3.0$ ) was dissolved in $700 \mathrm{cc}$ of $p$-xylene at boiling temperature under nitrogen atmosphere. Precipitates were obtained by slowly adding the hot solution into $3 l$ of methanol. The crystals were washed by acetone several times and dried at $50^{\circ} \mathrm{C}$ under vacuum. This curdy precipitate of HDPE was used for extrusion and also for further preparation of solution-grown crystals. The latter was precipitated from $0.03-, 0.10-$, and $0.25-\%$ solutions in $p$-xylene kept at $80^{\circ} \mathrm{C}$ for $20 \mathrm{~h}$, then slowly cooled to $<50^{\circ} \mathrm{C}$ (these crystals are referred to as SGC-0.03, SGC0.10 , and SGC-0.25, respectively). Two different samples, i.e., randomly oriented crystal aggregates and oriented (single) crystal mats, were prepared for extrusion of solution grown (single) crystals. For preparation of randomly oriented crystal aggregates, the crystal suspension was filtered to remove most of the solvent. The wet crystal aggregates were suspended in acetone to exchange xylene with the more volatile solvent, and then filtered again. These crystal aggregates were dried at room temperature for one day and then at $50^{\circ} \mathrm{C}$ under vacuum for one week. No weight loss was observed on prolonged drying, indicating the complete removal of solvents. Oriented mats of solution-grown crystals were prepared by slowly filtering the crystal suspension until most of the solvent was drained off, then more rapidly to improve the packing and orientation of the lamellar crystals.

\section{Crystalline-State Extrusion}

Two different techniques were used for the crystalline-state extrusion of solution-grown crystals.

Technique I. For the extrusion of randomly oriented crystals, a well-dried powder of solutiongrown crystal aggregates was packed into a reservior of an Instron capillary rheometer held at $90^{\circ} \mathrm{C}$ by applying a pressure of $0.23 \mathrm{GPa}$ for $3 \mathrm{~h}$. When extrusion was performed on these billets at $>90^{\circ} \mathrm{C}$, the temperature was raised under high pressure $(0.23$ $\mathrm{GPa})$ to reduce annealing of the initial morphology. The bulk density of these crystalline-state molded billets was slightly lower than that of meltcrystallized billets, indicating the presence of voids. After extrusion, however, the density of unoriented parts of the billet usually increased up to or sometimes exceeded that of the melt-crystallized billets suggesting that any initially trapped air was displaced before deformation at the entrance zone of the extrusion die.

Technique II. For the extrusion of oriented mats of solution-grown crystals, the coextrusion technique $^{20}$ recently developed in this laboratory was used. A melt-crystallized billet of HDPE was prepared by compression molding in vacuo with a specially designed apparatus. The billet was split longitudinally into halves, having smooth surfaces. One or more mats of solution-grown oriented mat(s) were placed between the split billet halves and the assembly coextruded. Both I- and II-type extrusions were performed through conical brass dies having an included entrance angle of $20^{\circ}$ and capillary length of $1 \mathrm{~cm}$.

\section{Tensile Modulus and Birefringence}

The modulus of the extrudate along the fiber axis was measured at room temperature on an Instron testing instrument, Model TTM, equipped with an Instron strain gage extensometer $(10 \mathrm{~mm})$. The modulus was determined at a strain rate of $\sim 3 \times 10^{-5} \mathrm{~s}^{-1}$ from the tangent to the stress-strain curve at $0.1-\%$ strain.

The birefringence of the extrudates was measured using an Ehringhaus Calcite Rotary compensator with a Zeiss polarizing microscope and a white light source.

\section{RESULTS AND DISCUSSION}

Highly oriented, transparent and uniform fibers and films were prepared by utilizing extrusion techniques I and II. Since the main purpose of this paper is to report on the effects of the initial morphology on the extrusion behavior, the extrusion performed by technique I (direct extrusion of solution-grown ( $\sin$ gle) crystals of powder form) will be emphasized.

\section{Extrusion Behavior of Solution-Grown Crystals and Melt-Crystallized Billets}

The morphology of solution crystals grown at $T_{\mathrm{c}}=80^{\circ} \mathrm{C}$ from $p$-xylene solutions of different concentrations were observed by electron microscopy. Most of the crystals grown from a $0.03-\%$ solution were well-defined, multilayer single crystals composed of mostly (110) fold domains and small (100) 
domains. Small overgrown crystals were frequently observed on the mother crystals. The number of regular single crystals decreased and generally the crystal morphology became more complex for increasing solution concentration. The term "single crystals" is nonetheless, used here for all crystals isothermally grown from dilute solutions $(0.03 \sim 0.25 \%)$. The morphology of curdy precipitates prepared by mixing a hot xylene solution into methanol was not clear; nevertheless it was presumed to be intermediate between the solution-grown crystal aggregates and melt-grown spherulitic samples.

In order to study the effect of initial morphology on the crystalline-state extrusion, single crystals grown from $0.03-\%$ (SGC-0.03), $0.10-\%$ (SGC-0.10), and $0.25-\%$ (SGC-0.25) solutions, and also curdy precipitates were extruded at $90^{\circ} \mathrm{C}$ and $0.23 \mathrm{GPa}$ through a die having an extrusion draw ratio (EDR) 30. A low temperature was chosen to minimize the annealing during/and after extrusion which would confuse the features of initial morphology on the structure and properties of the extrudates. Figure 1 shows the extrudate length $v s$. the extrusion time for single crystals and the curdy precipitates. With increasing length of the extrudate, the extrusion rate decreased, i.e., as the EDR increased and became constant (steady state extrusion) for the maximum EDR. Table I shows that there was no systematic change in the extrusion rate with initial morphology for a series of single-crystal samples. However, a pronounced effect of initial morphology is illustrated by the large difference in the steady-state extrusion rate of single crystals $(\sim 0.3 \mathrm{~cm} / \mathrm{min})$ and simple precipitates $(0.06 \mathrm{~cm} / \mathrm{min})$. Furthermore, under these extrusion conditions, the extrusion of meltcrystallized spherulitic morphology is very slow and stops before reaching a steady state (constant EDR). The aggregates formed from the $0.25-\%$ solution were used for extensive study. Figure 2 shows the extrudate length $v s$. time for these solution-grown crystals and for melt-crystallized spherulitic samples. The extrusion was performed at 70,90 and $110^{\circ} \mathrm{C}$ using a die having an entrance angle $20^{\circ}$ and an EDR of 45 , to study the effects of initial morphology. A merit of extrusion at low temperature is related to the efficiency of the extrusion draw evaluated by the modulus vs. EDR. Porter, Mead, ${ }^{14}$ and Shimada ${ }^{21}$ together showed that the efficiency of the draw increased with decreasing extrusion temperature in the range from an ambient melting point to $80^{\circ} \mathrm{C}$.

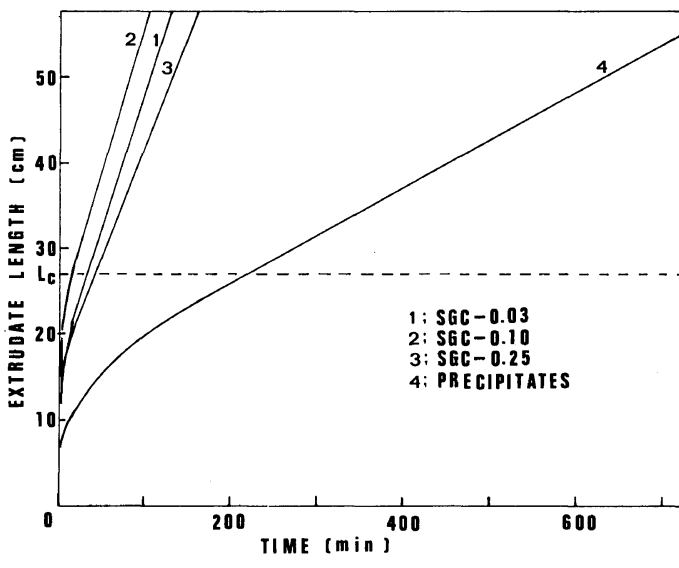

Figure 1. Extrudate length $v s$. time showing the effect of solution crystallization conditions on the extrusion behavior. Samples 1, 2, and 3 were crystallized at $80^{\circ} \mathrm{C}$ from $0.03-\%(1), 0.10-\%$ (2), and $0.25-\%$ (3) solutions in xylene. Sample 4 was precipitated by adding hot solution into methanol. Extrusion was performed at $90^{\circ} \mathrm{C}$ and 0.23 GPa through a die with EDR $30 . L_{\mathrm{c}}$ (dotted line) shows the critical length after which the extrusion reaches steady state (or constant EDR).

Table I. Extrusion of fibers from solution-grown crystals

\begin{tabular}{lcrcc}
\hline $\begin{array}{c}\text { Original } \\
\text { sample }\end{array}$ & $\begin{array}{c}\text { EDR, } \\
\text { const }\end{array}$ & $\begin{array}{c}T_{\text {ext }}, \\
{ }^{\circ} \mathrm{C}\end{array}$ & $\begin{array}{c}P_{\text {ext }}, \\
\mathrm{GPa}\end{array}$ & $\begin{array}{c}\text { Extrusion } \\
\text { rate, cm/min }\end{array}$ \\
\hline SGC-0.03 & 30 & 90 & 0.23 & 0.30 \\
SGC-0.10 & 30 & 90 & 0.23 & 0.35 \\
SGC-0.25 & 30 & 90 & 0.23 & 0.25 \\
Precipitates & 30 & 90 & 0.23 & $\sim 0.06$ \\
SGC-0.25 & 45 & 110 & 0.23 & $\sim 0.2$ \\
\hline
\end{tabular}

In Figure 2, it is clearly shown that solution-grown crystals extrude much faster than the spherulitic samples, thus reflecting their initial morphology. Another remarkable difference was the fracture mode of the extrudates. On extrusion of the spherulitic form, shear fractures appeared at about $45^{\circ}$ to the fiber axis at a critical EDR, varying with the extrusion conditions, (conditions for fracture onset are shown by arrows in Figure 2). The critical EDR for the fracture formation shifted to a higher EDR with increasing extrusion temperature and pressure in agreement with Shimada and Porter. ${ }^{21}$ In contrast to the extrusion of spherulitic materials, the extrudates of the solution-grown crystals seldom exhibited shear fracture. In contrast, the extrudates of 


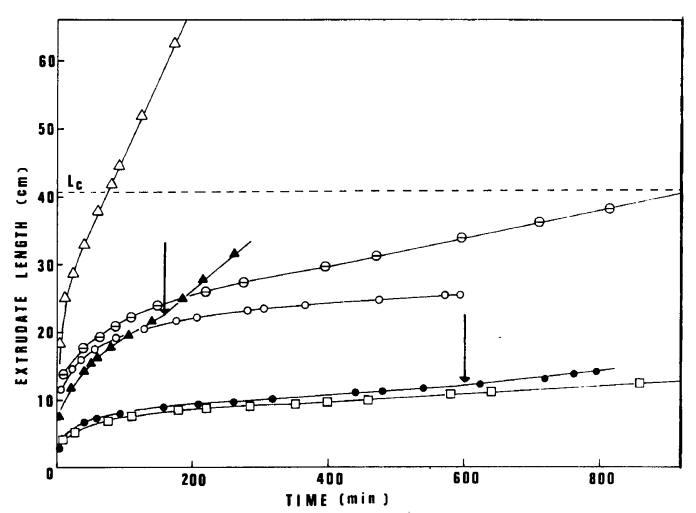

Figure 2. Extrusion behavior of solution-grown crystals and melt-crystallized solid plugs. Solution-grown crystals $\left(T_{\mathrm{c}}=80^{\circ} \mathrm{C}, c=0.25 \%\right)$ were extruded at $70^{\circ} \mathrm{C}$ $(\square), 90^{\circ} \mathrm{C}(\bigcirc$ and $\Theta)$ and $110^{\circ} \mathrm{C}(\triangle)$. Solid plugs were extruded at $90^{\circ} \mathrm{C}(\boldsymbol{O})$ and $110^{\circ} \mathrm{C}(\mathbf{\Delta})$. Extrusion was performed at $0.23 \mathrm{GPa}$ except $\ominus(0.34 \mathrm{GPa})$ through a die having EDR 45. The arrow indicates the first appearance of shear fracture.

solution-grown crystals frequently exhibited surface cracks running parallel to the extrusion direction. In order to examine the formation of such cracks, partially extruded billets with $1 \mathrm{~cm}$ of constantly drawn fiber (capillary part of a die) were removed from the die. The fact that no crack was found even at maximum EDR indicates that the surface cracks were formed after the extrudate came out of the capillary portion of the die, probably due to the difference in contraction between the surface and central regions of the extrudate caused by rapid cooling without compression force.

The effects of initial morphology on the extrusion rate and heterogeneity may be understood from the deformation mechanism. Three stages for deformation of spherulitic material have been proposed by Peterlin ${ }^{22}$ : stage I, deformation of spherulites combined with rotation and sliding of lamellae, and chain inclination within lamellae; stage II, fibril formation by chain slip and chain unfolding, and last; stage III, interfibrillar slip. The first stage is not important for explaining the observed effects of initial morphology, since it occurs only at low EDR. Although there is evidence suggesting the existence of irregular chain foldings on the surface of polyethylene single crystals, it is widely accepted that regular and adjacent re-entry chain foldings are the predominant mechanisms for single crystal grow- th. ${ }^{23,24}$ Thus, the number of inter- and intra-lamellar tie molecules must be smaller in solution-grown (single) crystals than in melt-crystallized spherulitic materials. Such a morphological feature suggests that fibril formation (stage II) due to chain unfolding readily proceeds with solution-grown crystals. With increasing EDR $(\sim 10)$ the third mechanism, interfibrillar slip, becomes important. As described above, the frequent appearance of cracks along the fiber axis of the extrudates of solution-grown crystals suggests that the interfibrillar connection is weaker than that of the spherulite extrudates, reflecting initial bondings between crystallites (lamellae). Weaker bondings between newly formed fibrils at stage II facilitate further deformation which proceeds mainly by interfibrillar slip at high EDR (stage III).

\section{Birefringence and Tensile Modulus}

The effect of initial morphology on the structure and properties of crystalline state extruded fibers

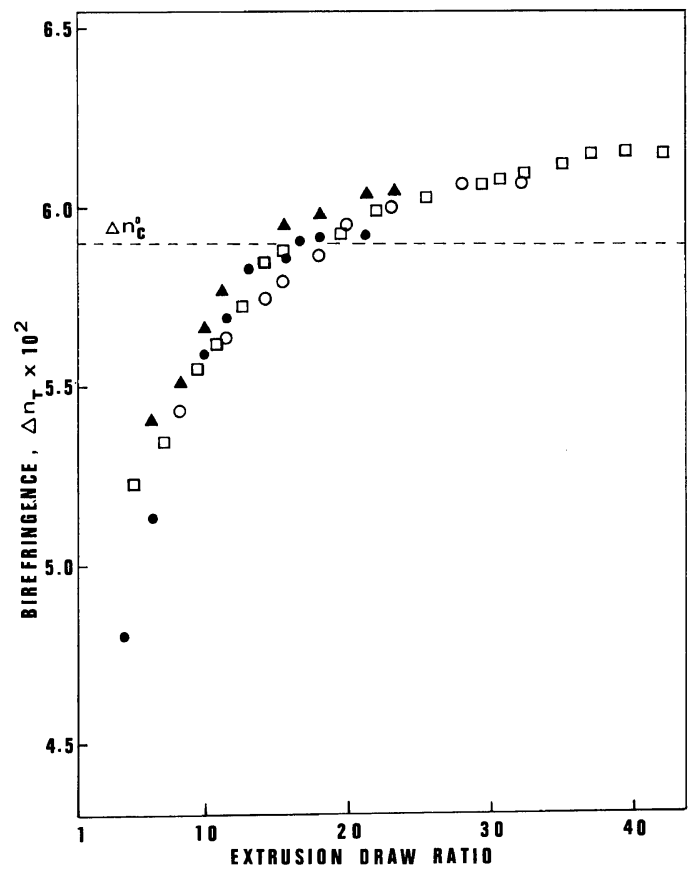

Figure 3. Birefringence vs. extrusion draw ratio for crystalline-state extruded fibers of solution-grown crystals and melt-crystallized solid plugs. Fibers from solution-grown crystals $\left(T_{\mathrm{c}}=80^{\circ} \mathrm{C}, c=0.25 \%\right)$ extruded at $90^{\circ} \mathrm{C}(\bigcirc)$ and $110^{\circ} \mathrm{C}(\square)$ and from solid plugs extruded at $90^{\circ} \mathrm{C}(\mathbf{O})$ and $110^{\circ} \mathrm{C}(\mathbf{\Delta}) . \Delta n_{\mathrm{c}}{ }^{\circ}$ (dotted line) indicates the birefringence value for a perfect polyethylene crystal. 
were evaluated by tensile modulus and birefringence. Figure 3 shows the total birefringence $\Delta n_{\mathrm{T}}$ as a function of EDR for the extrudates of solutiongrown crystals $\left(T_{\mathrm{c}}=80^{\circ} \mathrm{C}, c=0.25^{\circ} \%\right)$ and the meltcrystallized spherulitic samples. The extrusion was performed at $90^{\circ} \mathrm{C}$ and $110^{\circ} \mathrm{C}$ by a conical die with EDR 45 (and 51.5 respectively). The $\Delta n_{\mathrm{T}}$ rapidly increased with EDR and approached essentially plateau values of $0.059 \sim 0.062$ at EDR of $16 \sim 30$ with both changes depending on the initial morphology and extrusion temperature. The maximum value was higher than the intrinsic crystalline birefringence reported to be $\Delta n_{\mathrm{T}}{ }^{0}=0.0572 \sim 0.0585 .^{25,26}$ However, the general feature was almost the same irrespective of the above parameters, agreeing with previous work in this and other laboratories. ${ }^{7,27,28}$

Figure 4 shows the room temperature tensile modulus of the extrudates along the fiber axis determined by the tangent to the stress-strain cruve at $0.1-\%$ strain. The EDR varied continuously with the extrudate length. Both extrusions exhibited essentially the same modulus vs. EDR curve, which changed with extrusion temperature, but the maximum attainable EDR (and hence modulus) was higher for extrusion of solution-grown crystals than for melt-crystallized samples.

Ishikawa, et al., ${ }^{15,16}$ reported remarkable differences in the modulus and thermal stability of fibers drawn from single-crystal mats and melt-crystallized

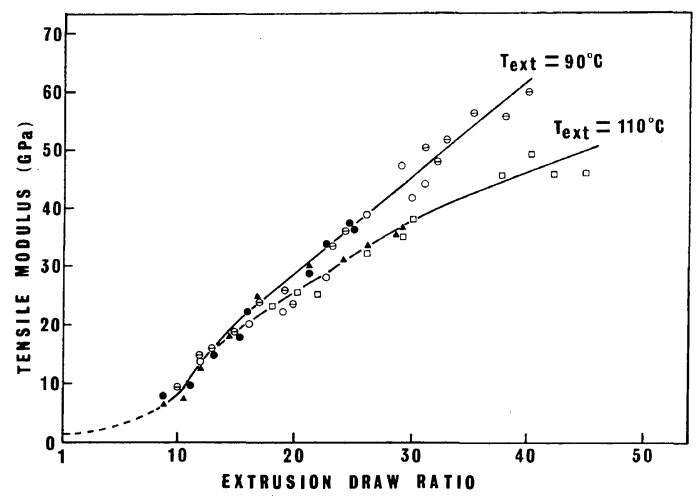

Figure 4. Tensile modulus $v s$. extrusion draw ratio of crystalline-state extruded fibers from solution-grown crystals and melt-crystallized solid plugs. Fibers from solution-grown crystals $\left(T_{\mathrm{c}}=80^{\circ} \mathrm{C}, c=0.25 \%\right)$ extruded at $90^{\circ} \mathrm{C}(\bigcirc$ and $\ominus)$ and $110^{\circ} \mathrm{C}(\square)$, solid plugs extruded at $90^{\circ} \mathrm{C}(\boldsymbol{O})$ and $110^{\circ} \mathrm{C}(\mathbf{\Delta})$. Extrusion was performed at $0.23 \mathrm{GPa}$ except $\ominus(0.34 \mathrm{GPa})$ through a die having EDR 45. morphology. However, the study was made on films drawn to necking, i.e., the samples had different DR characteristic for the initial morphologies $(D R=25$ for drawn mats of single crystals and 5 for drawn bulks). Thus, these results seem to reflect only the $\mathrm{DR}$, and are consistent with the present conclusion that the properties (birefringence and modulus) are unique functions of EDR irrespective of the initial morphology of the extrudates.

\section{CONCLUSION}

The initial morphology has a remarkable effect on the crystalline-state extrusion of HDPE but no appreciable effect on the properties (modulus and birefringence) of the resultant extrudates. Solutiongrown (single) crystals extruded at a rate faster than melt-crystallized spherulitic morphology. Precipitates obtained by mixing hot xylene solution into methanol extruded at an intermediate rate. Surface cracks were observed but shear fracture seldom occurred on single crystal extrudates. The maximum attainable EDR and hence the modulus was higher for the extrusion of single-crystal aggregates than for melt-crystallized spherulitic samples. However, there was no appreciable effect of initial morphology on the modulus vs. EDR, birefringence vs. EDR and transparency of the extrudates. This has major implications concerning the deformation mechanism. The results clearly show that intercrystalline networks and entanglements of the original sample remarkably affect the extrusion behavior and reduce the maximum achievable EDR (and hence modulus), but are not important in achieving efficient draw as evaluated by the physical properties $v s$. EDR. These results thus suggest that interfacial friction-adhesion between crystalline lamellae is a primary mechanism for drawing.

\section{REFERENCES}

1. J. H. Southern and R. S. Porter, J. Macromol. Sci.Phys., 4, 541 (1970).

2. H. Niikuni and R. S. Porter, J. Mater. Sci., 9, 389 (1974).

3. R. S. Porter, J. H. Southern, and N. Weeks, Polym. Eng. Sci., 15, 213 (1975).

4. K. Imada, T. Yamamoto, K. Shigematsu, and M. Takayanagi, J. Mater. Sci., 6, 537 (1971).

5. K. Imada and M. Takayanagi, Int. J. Polym. Mater., 2, 89 (1973). 
6. K. Nakayama and H. Kanetsuna, Kobunshi Kagaku, 30, 713 (1973).

7. T. Williams, J. Mater. Sci., 8, 59 (1973).

8. E. S. Clark and L. S. Scott, Polym. Eng. Sci., 14, 682 (1974).

9. T. Nakayama and N. Inoue, Trans. Jpn. Soc. Mech. Eng., 43, 3126 (1976).

10. C. J. Farrell and A. Keller, J. Mater. Sci., 12, 966 (1977).

11. A. G. Kolbeck and D. R. Uhlman, J. Polym. Sci., Polym. Phys. Ed., 15, 27 (1977).

12. G. Capacio and M. I. Ward, Polymer, 15, 233 (1974).

13. P. J. Barham and A. Keller, J. Mater. Sci., 11, 27 (1976).

14. W. T. Mead and R. S. Porter, to be published.

15. K. Ishikawa, K. Miyasaka, and M. Maeda, J. Polym. Sci., Part A-2, 7, 2029 (1969).

16. M. Maeda, K. Miyasaka, and K. Ishikawa, J. Polym. Sci., Part A-2, 8, 355 (1970).

17. W. O. Statton, J. Appl. Phys., 38, 4149 (1967).
18. P. J. Barham and A. Keller, J. Mater. Sci., 11, 27 (1976).

19. T. Kanamoto, A. E. Zachariades, and R. S. Porter, Polym. J., 11, 307 (1979).

20. P. D. Griswold, A. E. Zachariades, and R. S. Porter, presented, Stress-Induced Crystallization Symposium, Midland Macromolecular Institute, MI, August 1977.

21. T. Shimada and R. S. Porter, to be published.

22. A. Peterlin, J. Mater. Sci., 6, 490 (1971).

23. P. H. Geil, "Polymer Single Crystals," Interscience, New York, N.Y., 1963.

24. A. Keller, Rep. Prog. Phys., 31(2), 623 (1968).

25. R. S. Stein and F. H. Norris, J. Polym. Sci., 21, 381 (1956).

26. C. R. Desper, J. H. Southern, R. D. Ulrich, and R. S. Porter, J. Appl. Phys., 41, 4284 (1970).

27. W. T. Mead, C. R. Desper, and R. S. Porter, to be published.

28. K. Nakayama and H. Kanetsuna, J. Mater. Sci., 10, 1105 (1975). 